Military Technical College

Kobry El-Kobba

Cairo, Egypt

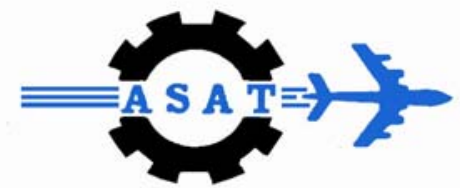

12-th International Conference

on

Aerospace Sciences \&

Aviation Technology

\title{
IMPROVING PERFORMANCE OF SPACE-TIME TURBO CODES WITH SHORT FRAMES IN NARROWBAND JAMMING
}

\author{
Moataz M. Salah
}

\begin{abstract}
This paper considers the performance enhancement of a space-time turbo codes processing system employing a combined turbo code and antenna transmission diversity scheme for direct sequence spread spectrum communication system.

Turbo codes have been shown to approach the Shannon limit for error correcting capability at low signal-to-noise ratios given large sized frames. Extensive research efforts have been examining ways to enhance the performance of turbo codes for short frames (e.g., voice transmission).

The proposed system is investigated over Rayleigh fading channels only and also in case of narrowband jamming environment in addition to Rayleigh fading channels.

This paper focuses on the implementation and performance of a modified turbo decoder for this model. Furthermore, the description of the concatenation of turbo code and direct sequence/spread spectrum with a space-time block codes are presented. Simulation results show that substantial benefits can be obtained by direct sequence spreading with space time coding the coded bits over Rayleigh fading and narrowband jamming environment.
\end{abstract}

KEYWORDS: Turbo Codes, Space-Time Codes, Spread Spectrum 


\section{INTRODUCTION}

Turbo codes [1] are an exciting channel coding scheme that achieve very low error rates $\left(10^{-5}\right)$ at signal-to-noise ratios close to Shannon limit. The encoder of turbo code is formed using a parallel concatenation of two or more component convolutional encoders. The key element of the encoder is the use of an interleaver which permutes the information sequence and then uses this as the input to the second component encoder.

In wireless communication systems such as cellular systems, information is typically sent in short frames (less than 300 bits). The size of the transmission frame limits the choice of error control codes. Convolutional codes are commonly employed in wireless systems. One of the advantages of convolutional codes is that their performance is independent of the frame size as long as the frame size is much larger than the constraint length of the code.

In 1993, a class of parallel concatenated convolutional codes (known as turbo codes) was introduced [1]. Extensive research efforts have been examining ways to enhance the performance of turbo codes for short frames (e.g., voice transmission).

The wireless channels are sensitive to time-varying impairments, such as multipath fading and interference. Achieving high data rates over wireless channels is a challenging task. In general, time-varying fading due to multipath propagation can be effectively combated by employing various diversity techniques, including time, frequency, and space diversity.

Space diversity is a well-known technique used to combat multipath fading in wireless communications channel. The multiple antennas on transmitter and receiver provide the space diversity. Recently, there has been a lot of work dedicated to the study of transmit diversity techniques [2-4]. A transmit diversity scheme for two transmit antennas and a simple decoding algorithm was introduced by Alamouti in [3] and generalized to an arbitrary number of transmit antennas as Space-Time Block Coding by Tarokh et al. in [4]. Space-time block codes are designed to achieve the maximum possible diversity order of $n_{T} . n_{R}$ for $n_{T}$ transmit and $n_{R}$ receive antennas under the constraint of having a simple decoding algorithm [4]. Furthermore, for a certain diversity advantage, they provide the minimum possible decoding delay. However, space-time block codes are not designed to achieve an additional coding gain. Therefore, space-time block codes should be concatenated with an outer code like turbo codes which provides a significant coding gain.

Also, wireless channels in a hostile environment are subject to interference and jamming. Direct sequence spread spectrum (DS/SS) signals are required to overcome the sever levels of the interference and jamming that are encountered in the transmission of signals such as narrowband jamming signals. In this research, we apply turbo codes to DS/SS system to improve the performance over jamming signals and then combined with transmit diversity to combat the deep fades in the wireless channel.

The paper is organized as follows. Section II, describes the basic principles of turbo codes and space time block coding. Section III, illustrates the details of the proposed concatenation model. Section IV, presents the simulation results of the proposed model and show the performance over Rayleigh fading and jamming channels. Conclusions are summarized in Section V. 


\section{OVERVIEW TURBO CODES AND SPACE-TIME CODES}

In this section, we first give briefly review of the basic principles of turbo codes. We will then review the space time coding technique.

\section{II.1 Turbo Codes}

The block diagram of the turbo encoder is shown in Figure 1. Two identical recursive systematic convolutional (RSC) component codes of rate $1 / 2$ which are separated by an interleaver form the turbo encoder. Thus, the same information sequence is encoded twice but in different orders. The output sequence from the upper encoder and from the lower encoder are multiplexed and optionally punctured.

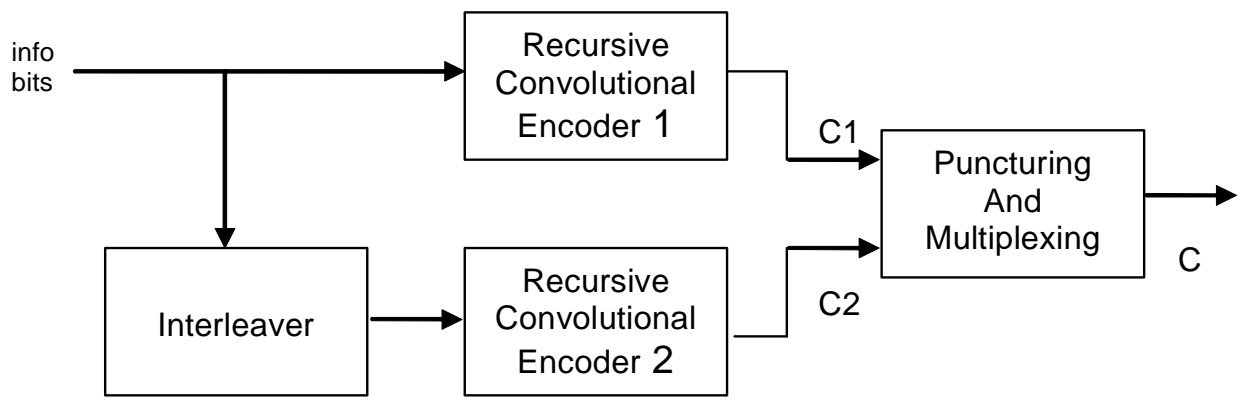

Figure 1. Turbo Code Encoder.

Note that turbo decoder does not perform maximum likelihood decoding directly, but attempts to achieve maximum likelihhod decoding in an iterative way. The original turbo decoder [1] used two maximum apostriori probability (MAP) algorithm decoders. There are other less complex algorithms that can be used in place of the MAP algorithm for each decoder such as SOVA (soft output Viterbi algorithm) [ 5 ] and Max-log MAP [6 ].

\section{Il.2 Space-Time bolck coding}

Space-time coding (STC) is an open loop transmission scheme that was introduced in [2-4]. In STC, joint design of channel coding and modulation is done to create efficient transmission techniques which improve system performance by providing both the diversity advantage of multiple transmit antennas and coding gain.

The author in [3] introduced a simple open loop scheme which provides a transmit diversity gain similar to that obtained by using Maximal Ratio Combining (MRC) in receive diversity systems. This scheme was proposed for two transmit antennas. Symbols transmitted from these antennas are encoded in space and time in a simple manner to ensure that transmissions from both the antennas are orthogonal to each other. This would allow the receiver to decode the transmitted information with very little additional computational complexity even if it has a single antenna element. Using the theory of orthogonal designs, the simple scheme proposed in [3] was generalized to systems with more than two transmit antennas [4]. These generalized schemes were called space-time block codes (STBC). 
A multi-antenna communication system, equipped with $n_{T}$ transmit antennas and receive $n_{R}$ antennas is shown in Figure 2. The input to a space-time block encoder is a block of $K$ complex symbols $x_{i}, i=1, \ldots, K$, where $x_{i}$ are elements of a higher order modulation constellation, e.g. M-PSK. The space-time block encoder maps the input symbols on entries of a $p \times n_{T}$ matrix $G$, where $p$ is the successive transmission instants and $n_{T}$ is the number of transmit antennas. The entries of the matrix $G$ are the $K$ complex symbols $x_{i}$, the conjugates $x_{i}^{*}$ and linear combinations of $x_{i}$ and $x_{i}^{*}$.

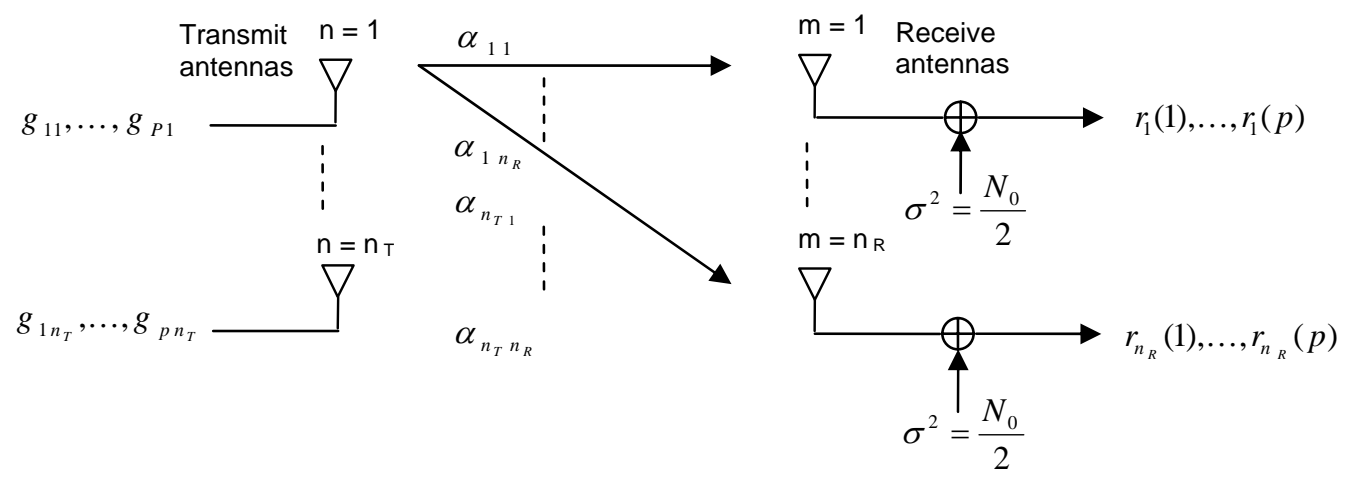

Figure 2. Channel matrix of space time block code.

The $p \times n_{T}$ matrix $G$ which defines the space time block code is a complex generalized orthogonal design as defined in [4], which means that the columns of $G$ are orthogonal. An Example for $n_{T}=2$ is the complex generalized orthogonal design

$$
\begin{aligned}
& \text { (Space) } \\
& \text { Antenna } 1 \text { Antenna } 2
\end{aligned}
$$

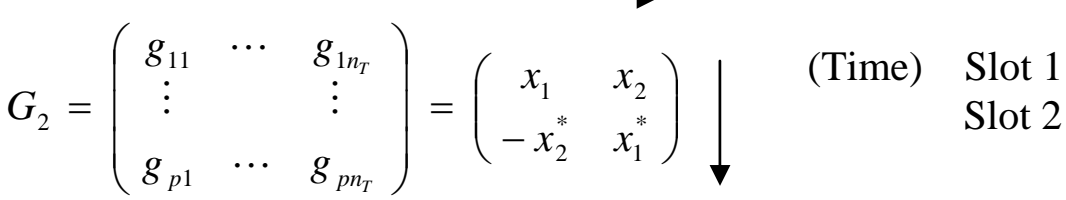

The associated encoding and transmission process is shown in Table (1).

Table 1. The encoding of G2 space-time block code.

\begin{tabular}{|l|l|l|}
\hline \multirow{2}{*}{ Time Slot } & Antenna \\
\cline { 2 - 3 } $\mathrm{T}$ & 1 & 2 \\
\hline 1 & $x_{1}$ & $x_{2}$ \\
\hline 2 & $-x_{2}^{*}$ & $x_{1}^{*}$ \\
\hline
\end{tabular}

At any given time instant $T$, two signals are simultaneously transmitted from the antennas, For example, in the first time slot associated with $T=1$, signal $x_{1}$ is transmitted from antenna 1 and simultaneously signal $x_{2}$ is transmitted from antenna 2. In the next time slot corresponding to $T=2$ signals $-x_{2}^{*}$ and $x_{1}^{*}$ (the conjugates of symbols $x_{1}$ and $x_{2}$ respectively ) are simultaneously transmitted from antennas 1 and 2 respectively. 
$G_{2}$ is the scheme proposed in [3]. For more designs we refer to [4]. Generally all symbols in the same row of $G$ are transmitted simultaneously from $n_{T}$ different antennas. All entries in the same column of $G$ are transmitted from the same antenna in $p$ successive transmission instants. Given the number $K$ of symbols to be transmitted the rate $R$ should be maximized.

$$
R=\frac{K}{p}
$$

In case of $G_{2}$ the rate $R=\frac{2}{2}=1$, this means that the two symbols are transmitted in two time slots.

\section{PROPOSED SYSTEM MODEL}

The proposed system block diagram is shown in Figure 3. The information sequence inputting the system is encoded using turbo code. The output is Binary Phase Shift Key (BPSK) modulated. The modulated output is DS/SS modulated. Prior to transmission, the modulated sequence is formatted by the space-time (ST) block encoder. ST blocks could be two, three or four transmit antennas and one, two, three or four in the in the reconceived site.

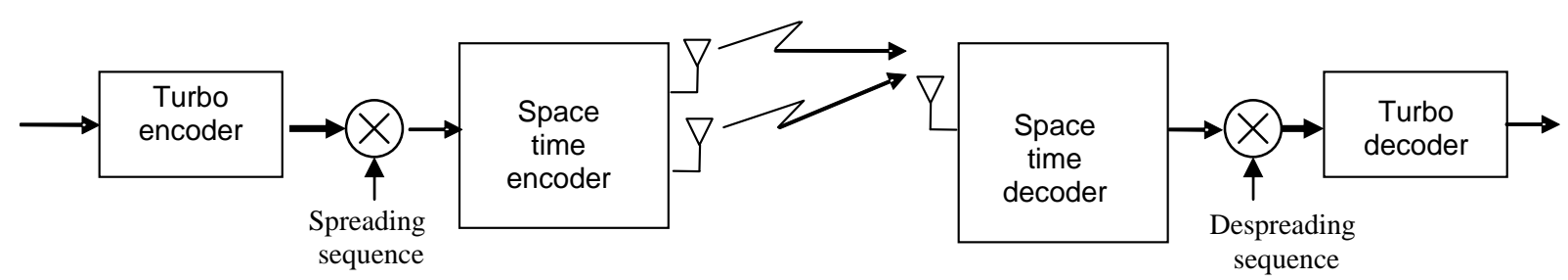

Figure 3. The Proposed concatenation model.

Turbo code decoder uses soft values in decoding the transmitted symbols, so we need to derive a soft output symbol by symbol (MAP) from the out of space time decoder. Then we will use this new soft values to modify the turbo decoder metric for decoding operation.

\section{Soft values derivation:}

At the receiver we can use optional antenna diversity using $n_{R}$ receive antennas. The orthogonality of the columns of $G$ allows us to derive a simple MAP decoding rule by decoupling the signals transmitted from different antennas when the channel doesn't change for the duration of $p$ consecutive symbols.

We consider a flat fading matrix channel as shown in Figure 2, where $\alpha_{n m}$ denotes the complex fading factor from transmit antenna $n$ to receive antenna $m$.

At each time instant $t=1, \ldots, p$ and each receive antenna $m=1, \ldots, n_{R}$ we observe a superposition $r_{m}(t)$ of the signals transmitted from the $n_{T}$ antennas perturbed by additive white Gaussian noise with a two sided spectral power density of $\sigma^{2}=\frac{N_{0}}{2}$ per dimension. 
Let $r(t)=\left(r_{1}(t), \ldots, r_{n_{R}}(t)\right)$ denotes the vector of received signals at time $t$ and let $c=\left(c_{1}, \ldots, c_{K}\right)$ denotes the sequence of real valued symbols, where $c_{i} \in\{0,1, \ldots, M-1\}$, before the mapping on complex constellation symbols $x_{i}$. Then using Bayes' rule, the aposteriori probability of the transmitted sequence $c=\left(c_{1}, \ldots, c_{K}\right)$ given the received sequence $(r(1), \ldots, r(p))$ can be expressed as follows [7 ]:

$$
P\left(c_{1}, \ldots, c_{K} \mid r_{1}, \ldots, r_{p}\right)=\text { const } \cdot P\left(r_{1}, \ldots, r_{p} \mid c_{1}, \ldots, c_{K}\right) \cdot P\left(c_{1}, \ldots, c_{K}\right)
$$

After some mathematical manipulations

$$
P\left(r_{1}, \ldots, r_{p} \mid c_{1}, \ldots, c_{K}\right)=\text { const } \cdot e^{-\frac{1}{2 \sigma^{2}} \sum_{m=1}^{n_{R}} \sum_{t=1}^{p}\left|r_{m}(t)-\sum_{n=1}^{n_{T}} g_{t n} \alpha_{n m}\right|^{2}}
$$

$P\left(c_{1}, \ldots, c_{K}\right)$ is an apriori information which can be obtained from knowledge of the source statistics or from the aposteriori information of another decoder. Since the $c_{i}$ are statistically independent, the apriori term in equation 3 can be factorized as follows:

$$
P\left(c_{1}, \ldots, c_{K}\right)=\prod_{i=1}^{K} P\left(c_{i}\right)
$$

Since all symbols $g_{t n}$ in one column of $G$ are transmitted from the same antenna and therefore are multiplied with the same fading factor $\alpha_{n m}$ and since the columns in $G$ are orthogonal we obtain for the logarithm of equation 3 :

$\ln P\left(r_{1}, \ldots, r_{p} \mid c_{1}, \ldots, c_{K}\right)=$

$$
\text { const }-\frac{1}{2 \sigma^{2}} \sum_{m=1}^{n_{R}}\left\{\sum_{t=1}^{p}\left[-r_{m}(t) \sum_{n=1}^{n_{T}} g^{*}{ }_{t n} \alpha^{*}{ }_{n m}-r_{m}(t) \sum_{n=1}^{n_{T}} g_{t n} \alpha_{n m}\right]+\sum_{t=1}^{p} \sum_{n=1}^{n_{T}}\left(\left|g_{t n}\right|^{2}\left|\alpha_{n m}\right|^{2}\right)\right\}
$$

Each entry $g_{t n}$ of $G$ corresponds to a certain symbol $c_{i}$ and each additive term in equation 5 contains only a single $g_{t n}$.

Consequently, we can decouple the aposteriori probabilities for the symbols $c_{i}$ and using equation 2, 3 and 5 a simple expression for the aposteriori probabilities of each symbol $c_{i}$ can be obtained as follows:

$$
\ln P\left(c_{i} \mid r_{1}, \ldots, r_{p}\right)=\text { const }+\ln P\left(r_{1}, \ldots, r_{p} \mid c_{i}\right)+\ln P\left(c_{i}\right)
$$

For example for the space-time block code $G_{2}$ with $n_{T}=2, K=2, p=2$ we obtain the aposteriori probabilities (soft output) is given by:

$\ln P\left(c_{1} \mid r(1), r(2)\right)=$

$$
\text { const }-\frac{1}{2 \sigma^{2}}\left|\left[\sum_{m=1}^{n_{R}}\left(r_{m}(1) \alpha_{1 m}^{*}+r_{m}(2)^{*} \alpha_{2 m}\right)\right]-x_{1}\right|^{2}-\underbrace{\frac{1}{2 \sigma^{2}}\left(-1+\sum_{m=1}^{n_{R}} \sum_{n=1}^{2}\left|\alpha_{n m}\right|^{2}\right)\left|x_{1}\right|^{2}}_{\text {Cons tant for } M-P S K \text { modulation }}+\ln P\left(c_{1}\right)
$$




$$
\begin{aligned}
& \ln P\left(c_{2} \mid r(1), r(2)\right)= \\
& \quad \text { const }-\frac{1}{2 \sigma^{2}}\left|\left[\sum_{m=1}^{n_{R}}\left(r_{m}(1) \alpha_{2 m}^{*}-r_{m}(2)^{*} \alpha_{1 m}\right)\right]-x_{2}\right|^{2}-\underbrace{\frac{1}{2 \sigma^{2}}\left(-1+\sum_{m=1}^{n_{R}} \sum_{n=1}^{2}\left|\alpha_{n m}\right|^{2}\right)\left|x_{2}\right|^{2}}_{\text {Cons tant for } M-P S K \text { modulation }}+\ln P\left(c_{2}\right)
\end{aligned}
$$

We will use the middle term to modify the turbo decoder metric. The constants can be omitted.

\section{SIMULATION RESULTS:}

This section contains the simulation results which illustrate the performance of binary turbo codes in combination with space time coding scheme and direct sequence/spread spectrum (DS/SS). The performance is measured in terms of the bit error rate (BER) as a function of the bit energy to noise ratio $E_{b} / N_{0}$. fot turbo codes, the $1^{\text {st }}$ Component code is terminated and the $2^{\text {nd }}$ is left open. A random interleaver with odd-even separation [8] is used. The output stream is BPSK modulated and sent through two transmit antennas and one antenna at the receiver site. A LOG-MAP decoder [6] with 8 iterations is employed at the receiver. A LOGMAP decoder is similar to a maximum a-priori probability (MAP) decoder, but simpler since only addition and subtractions are performed [6].

We scale down the transmit power from each antenna to maintain a unity total power with two identical component codes 4-state (1,5/7 octal) and different coding rates. The frame length used in all experiments is 200 bits.

In any given transmission period two signals are transmitted simultaneously from two transmit antennas. The encoder takes two symbols $x_{1} x_{2}$ at a time. Signal $x_{1}$ is transmitted from the first transmit antenna while signal $x_{2}$ from the second one. In the next transmission instance, signal $-x_{2}{ }^{*}$ is transmitted from the first transmit antenna and signal $x_{1}{ }^{*}$ from the second one, where $x_{i}{ }^{*}$ is the complex conjugate of $X_{i}, \mathrm{i} \in\{1,2\}$. Assuming that one receive antenna is employed and that the fading coefficients are constant across two consecutive symbols, the channel fading gains $h_{1}(t)$ and $h_{2}(t)$, for the first and the second transmit antennas, respectively, can be expressed as follows:

$$
\begin{aligned}
& h_{1}(t)=h_{1}(t+T)=h_{1}=\left|h_{1}\right| e^{j \theta_{1}} \\
& h_{2}(t)=h_{2}(t+T)=h_{2}=\left|h_{2}\right| e^{j \theta_{2}}
\end{aligned}
$$

Where $\left|h_{i}\right|, i \in\{1,2\}$ is the channel gain from transmit antenna $i$ to the receive antenna, $T$ is the symbol duration, and $\theta_{i}$, is the channel phase shift from transmit antenna $i$ to the receive antenna.

At the receiver, a combiner [3] forms the following combined signals, $r(1)$ and $r(2)$ : 


$$
\begin{aligned}
& r(1)=\left(\left|h_{1}\right|^{2}+\left|h_{2}\right|^{2}\right) x_{1}+h_{1}^{*} n_{1}+h_{2} n_{2}^{*} \\
& r(2)=\left(\left|h_{1}\right|^{2}+\left|h_{2}\right|^{2}\right) x_{2}-h_{1} n_{2}^{*}+h_{2}^{*} n_{1}
\end{aligned}
$$

Where $r(1)$ and $r(2)$ the received signals at time $t$ and $t+T$, respectively. The noise added $n_{i}, i \in\{1,2\}$ is a random variable representing a sample of additive white Gaussian noise. We will substitute $r(1)$ and $r(2)$ for calculations of the modified metric of turbo code decoder.

Assuming the channel is subject to Rayleigh fading with fading coefficients being independent and identically distributed random variables (perfectly interleaved narrow-band or non-dispersive Rayleigh fading channel). It is assumed that the receiver had a perfect estimate of the channels' fading amplitudes.

Figure 4 shows the performance comparison of the turbo code with, turbo code with space time transmit diversity with two transmit antennas, and turbo codes with direct sequence spread spectrum (DS/SS) with processing gain of 30 and use transmit diversity at final stage with two transmit antennas. The frame length used is 200 bits, the code rate is $1 / 2$. The performance curves show that the turbo codes with spread spectrum and transmit diversity is superior in performance as compared with other curves. As the spreading length increases the performance of the proposed system is enhanced and this is shown in Figure 5. This figure, using the same parameters used in Figure 4, shows the comparison of the proposed system without spreading and with two different spreading lengths, 20 and 50. The figures illustrate that as spreading length increases the performance is enhanced. Figures 6 and 7, for code rate $1 / 3$ and $1 / 2$ respectively, illustrate the performance for 2,46 and 8 iterations, show the importance of having a sufficient number of iterations at the decoder. The performance is significantly increased with four iterations compared with the two iterations. The improvement gained diminishes as the number of iterations increases from 6 to 8 . 


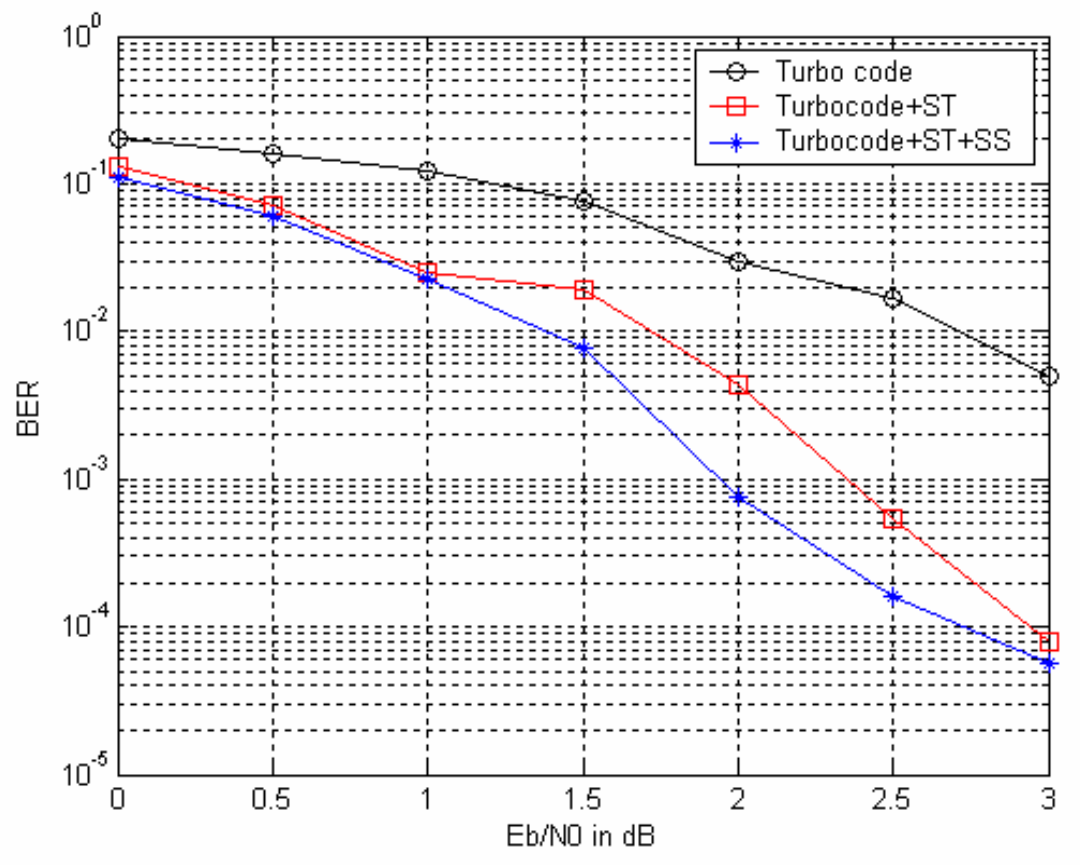

Figure 4. Performance comparison of turbo code, combined turbo code and space time (ST), and combined turbo code and space time coding with DS/SS.

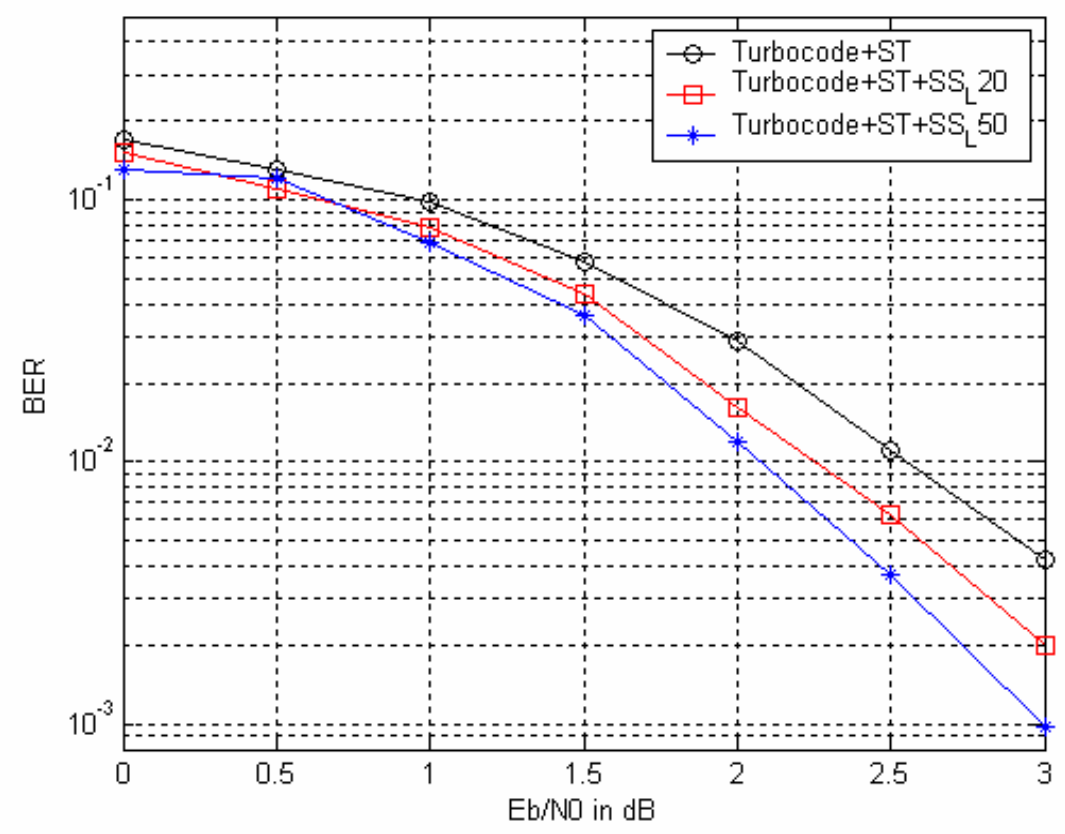

Figure 5. Effect of spreading processing gain on the performance of the proposed model. 


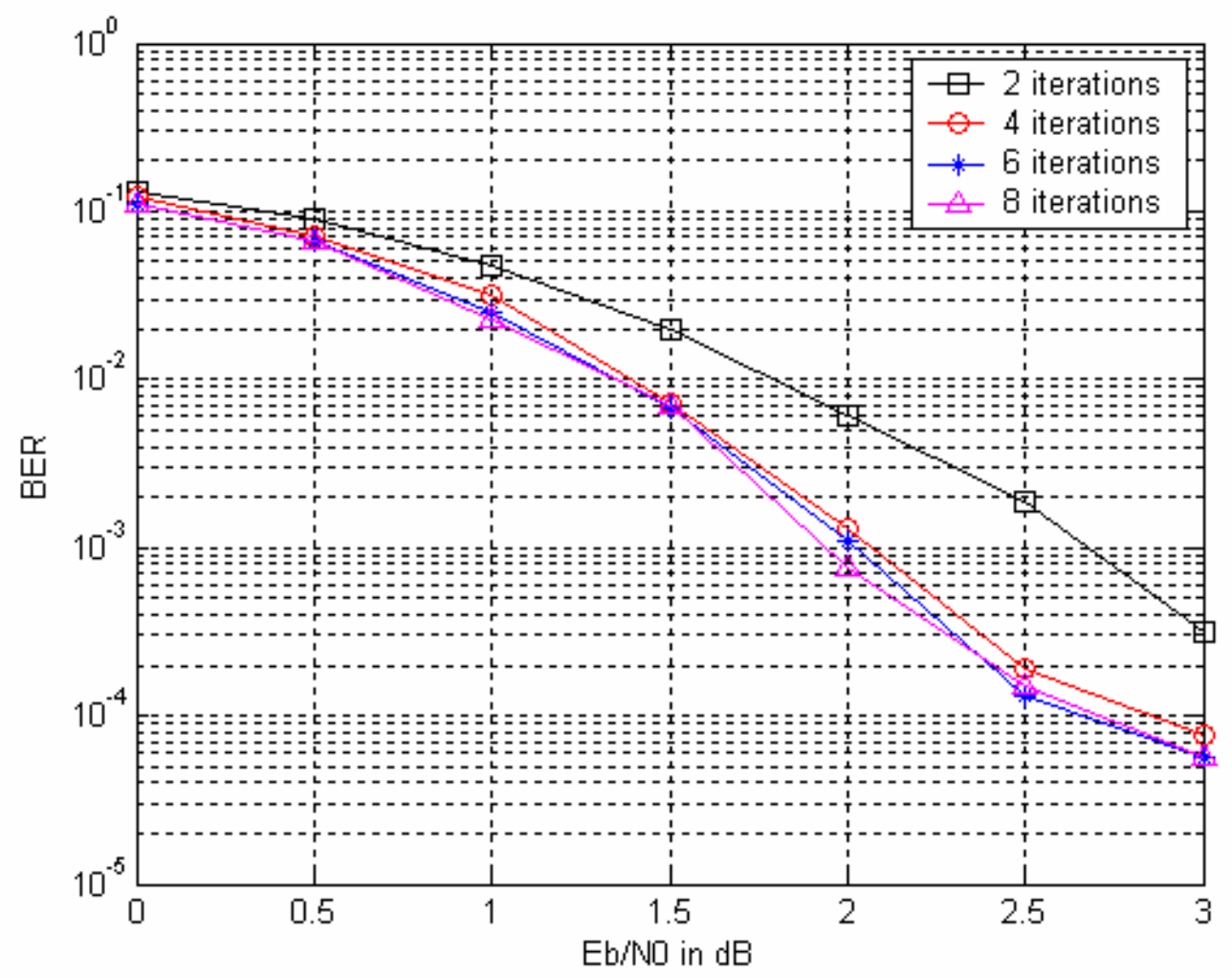

Figure 6. Effect of the number of iterations for rate 1/3.

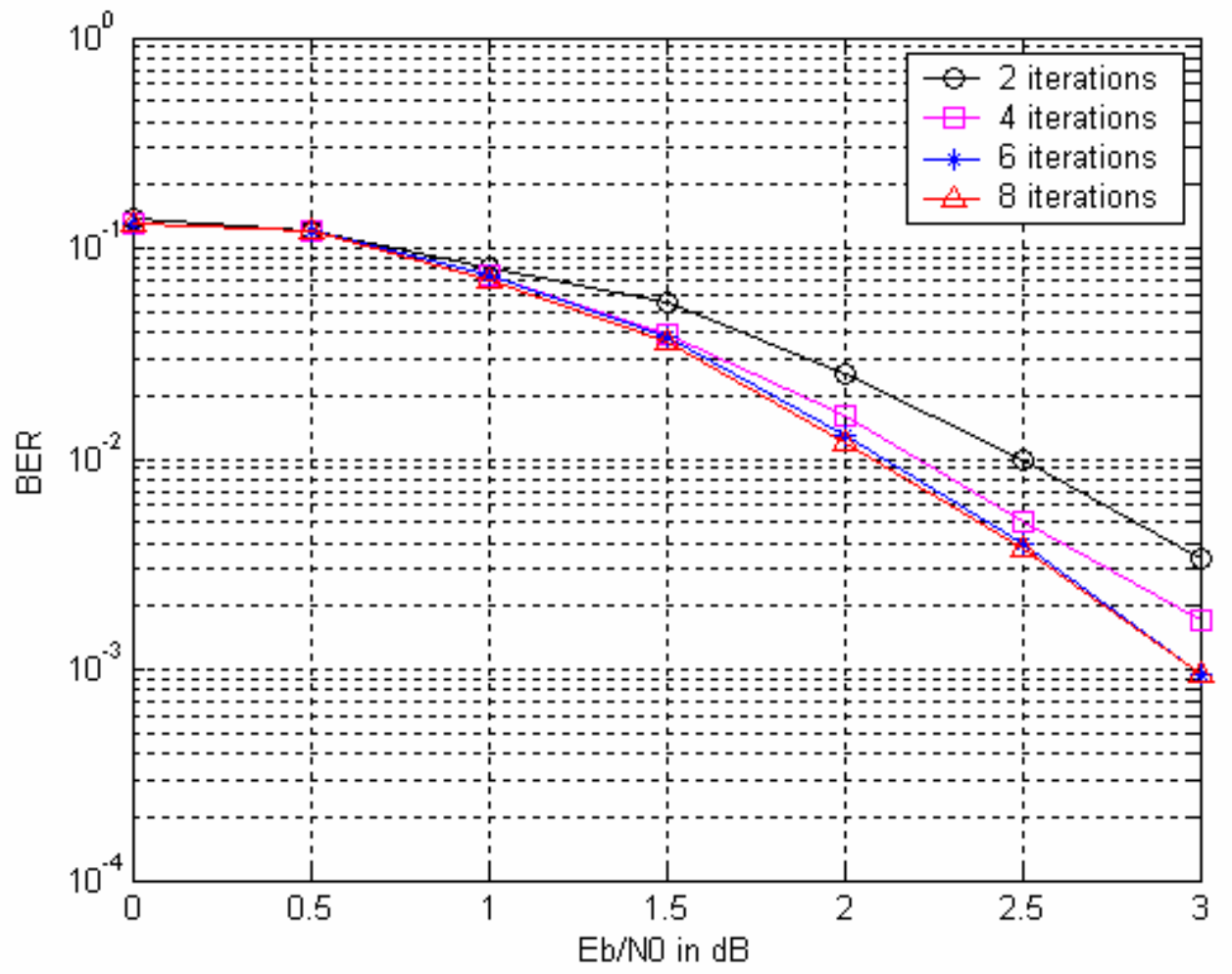

Figure 7 . Effect of the number of iterations for rate 1/2. 


\section{Narrowband Jamming effect:}

The proposed system performance is also investigated in narrowband interfering environment, known also as tone jammer. Let a sinusoidal interfering signal is given by [9]:

$$
i(t)=A_{j} \cos 2 \pi f_{j} t
$$

Where $f$ is a frequency within the bandwidth of the transmitted signal, with $P_{j}=A_{j}^{2} / 2$ average power. The power in the interfering signal is reduced by an amount equal the processing gain, $L_{\text {chip }}$, with using diect sequence/ spread spectrum. We apply this signal and added to the received signal to the proposed system model and subject to Rayleigh fading channel. Figures 8 and 9 show the performance of the of the proposed model with code rate $1 / 3$. Figures 8 and 9 show the performance in case of jamming amplitude, $A_{j}=3$ and $A_{j}=7$ for different processing gain values. As the processing gain increases, the performance of the proposed model is enhanced and more processing gains is required to mitigate higher values of the jamming power.

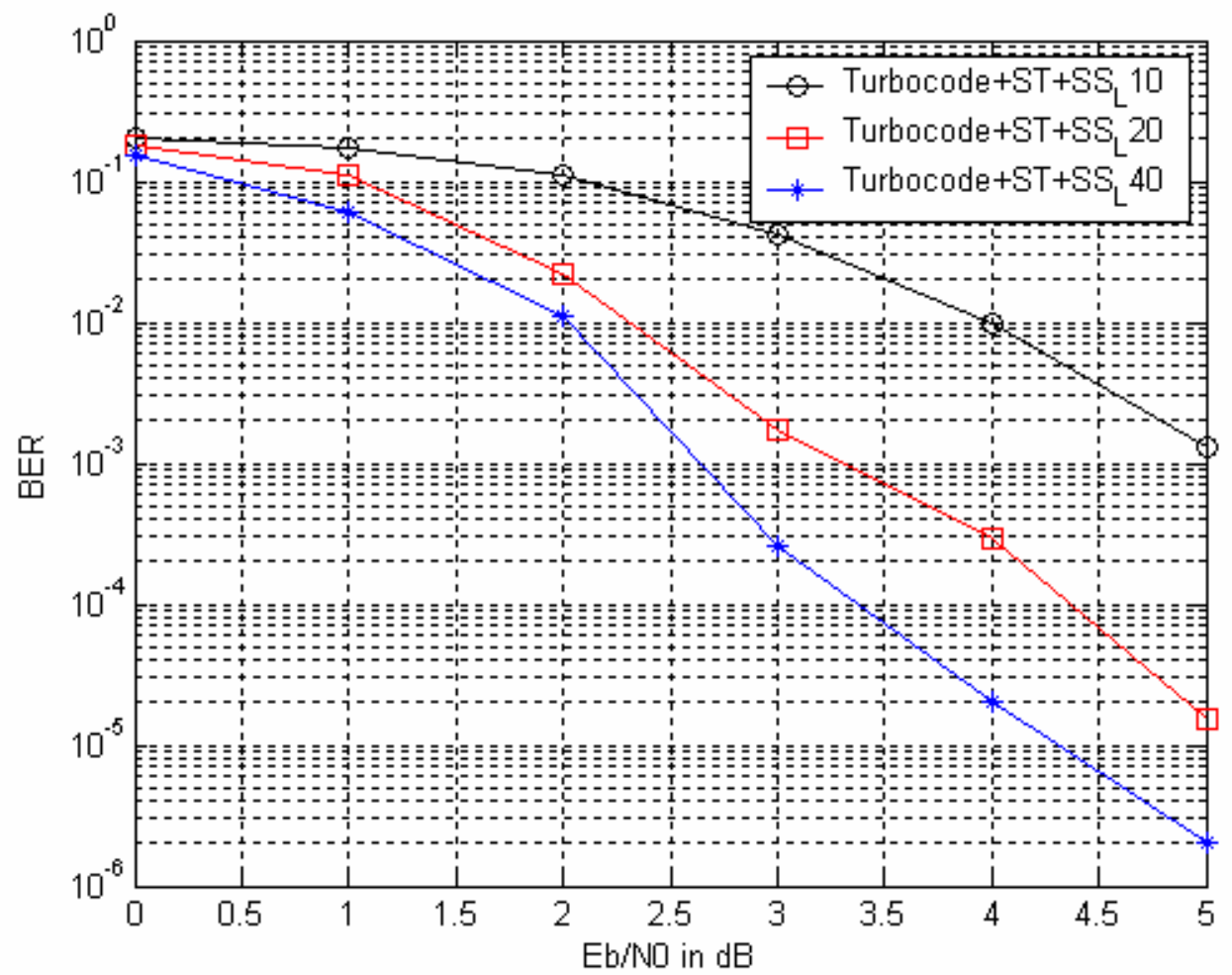

Figure 8. Performance of the proposed model with $A j=3$ and different processing gains. 


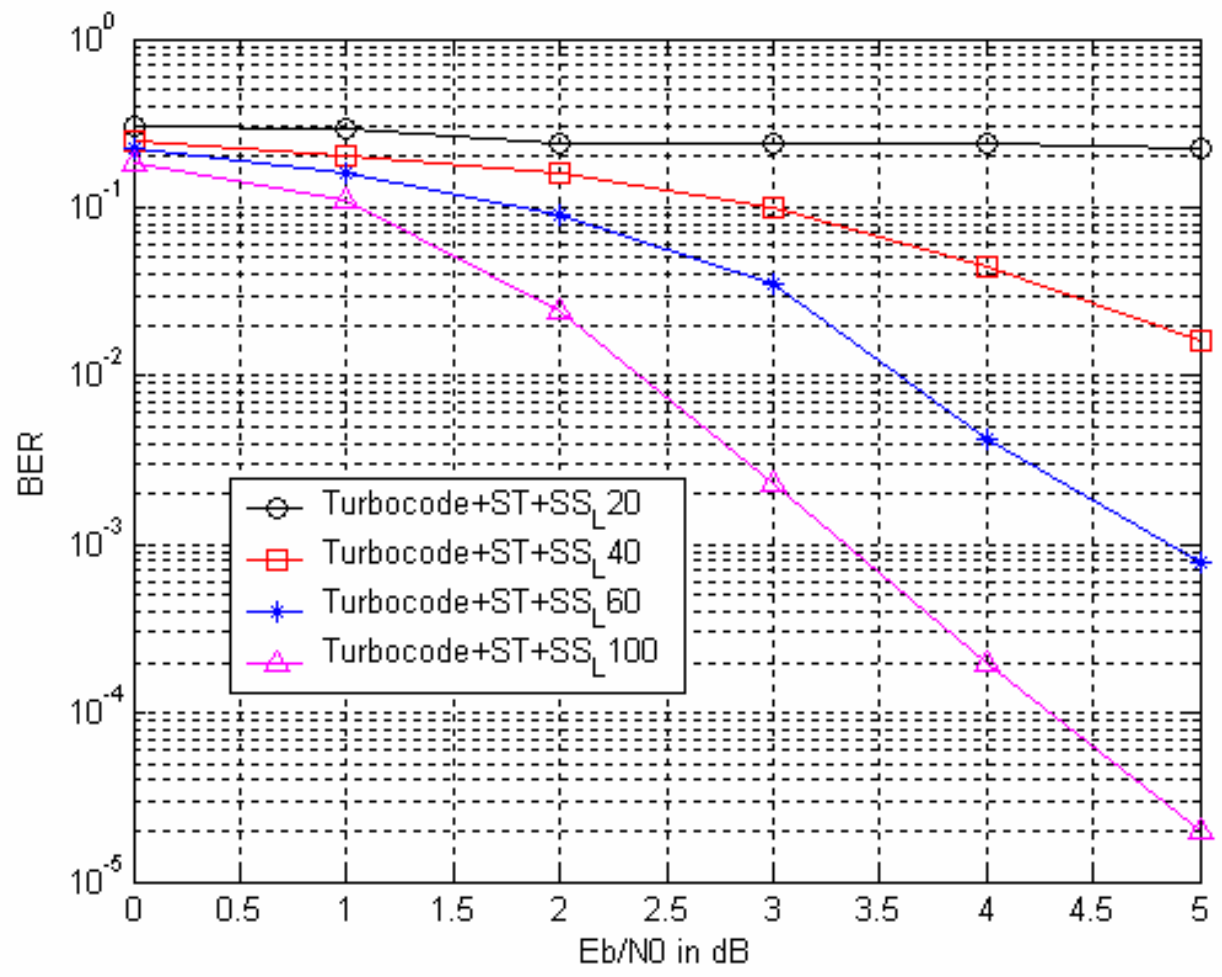

Figure 9. Performance of the proposed model with $A j=7$ and different processing gains.

\section{CONCLUSIONS}

In this paper, a concatenation of turbo codes, direct sequence spread spectrum, and transmit diversity is investigated for short frame lengths. The proposed system is investigated over Rayleigh fading channels only and also in case of narrowband jamming environment in addition to Rayleigh fading channels. The description of the concatenation of turbo code and direct sequence/spread spectrum with a space-time block codes were presented. Turbo code decoder was modified to handle this concatenation. The curves show that transmit diversity mitigate the effect of the fading in the channel and the direct sequence spread spectrum mitigates the existence of jamming, as the processing gain increased the degradation in the performance due to the existence of the jamming signal is decreased.

\section{REFERENCES}

[1] C. Berrou, A. Glavieux, and P. Thitimajshima, "Near Shannon limit error-correcting coding and decoding: Turbo-codes," in Proceedings of ICC'93, pp. 1064-1070, 1993.

[2] Jibing Wang, Marvin K. Simon "On the Performance of Space-Time Codes Over Spatially Correlated Rayleigh Fading Channels",IEEE Transactions on Communications, vol. 52, no. 6, June 2004. 
[3] S. M. Alamouti, "A simple transmit diversity technique for wireless communications," IEEE J.Selected Areas in Communications, vol. 16, pp. 14511458, Oct. 1998.

[4] V. Tarokh, H. Jafarkhani, and A. Calderbank, "Space-time block codes from orthogonal designs", IEEE Trans. Inform. Theory, vol. 45, pp. 1456-1467, July 1999.

[5] J. Hagenauer and P. Hoeher, "A Viterbi algorithm with soft-decision outputs and its applications" in Proc. IEEE Global Telecommunications Conf., Dallas, TX, Nov. 1989, pp. 47.1.1-47.1.7.

[6] P. Robertson, E. Villebrun, and P. Hoeher, "A comparison of optimal and suboptimal MAP decoding algorithms operating in the log domain," in Proceedings of ICC'95, pp. 1009-1013, 1995.

[7] A. I. Mahroos, "Turbo Codes for Mobile and Satellite Communication Systems," M.Sc. thesis, Military Technical College, Cairo, 2006.

[8] A. Barbulescu and S. Pietrobon, "Interleaver design for turbo codes," IEE Electronics Letters, pp. 2107-2108,December 1994.

[9] J. G. Proakis, Digital Communications, New York: McGraw-Hill, $4^{\text {th }}$ ed., 2001. 